\title{
Propuesta de clasificación técnica de los sistemas de explotación de las BN1G de producción (núcleos) ${ }^{1}$
}

\author{
Nuria Castañeda Clemente*
}

\begin{abstract}
RESUMEN ABSTRACT
Se propone una clasificación de los sistemas de explotación de las $B N 1 G$ de producción a partir de las relaciones entre los distintos atributos que caracterizan a los núcleos. Esta clasificación se estructura en tres niveles. El primero discrimina los sistemas de explotación según su número de supenficies de talla. En el segundo nivel, la característica jerarquizadora es la relación geométrica entre superficie / plataforma o entre superficies de talla. El tercer y último nivel atiende a la dirección en la que se explotan estas superficies.

This work consists in a classification of the production BN1G (cores) exploitation systems, based on the relationship between the different characteristic core forms features. There are three levels of classification. The first one discriminate between exploitation systems by their number of debitage surfaces. On the second level the hierarchical feature is the geometrical relationship between the debitage surface and striking platform or between debitage surfaces. Third level is the direction on exploitation of surfaces.
\end{abstract}

* Laboratori d'Arqueologia i Ensenyament de la Universitat Autonoma de Barcelona.

Este trabajo ha sido posible gracias a la dirección del Dr. Rafael MORA TORCAL y a los medios del Laboratori d'Arqueologia i Ensenyament de la Universitat Autònoma de Barcelona. 
Los esquemas de talla pueden conocerse, al menos en su estadío final, a través de los negativos de extracciones que caracterizan las Bases Negativas de 1. a Generación (Carbonell, Guilbaud, Mora, 1983, Mora, Martínez, Terradas, 1992, Mora, 1994). Su morfología general no es tan importante como la relación que guardan entre sí los distintos atributos de cada núcleo. Estas relaciones tienen como punto de partida la dialéctica entre la plataforma de talla y la superficie de talla (Mora, 1994). La plataforma de talla es el plano sobre el que se produce la percusión que dará lugar a la extracción de una lasca. La fuerza proporcionada se transmite a través de la materia del nódulo guiándose por las zonas más resistentes de la superficie isostática las aristas-, provocando una fractura que sigue la forma geométrica del elipsoide de Lamé (Bertouille, 1989). Esta fractura, que constituye el desprendimiento de un producto de talla o lasca, se desarrolla en un plano distinto al de la plataforma de percusión. Este plano se denomina superficie de talla y ambos son interdependientes; es decir, no puede existir el uno sin el otro.

Se establece un primer nivel de clasificación atendiendo a la intencionalidad de la explotación que tiene lugar en una BN1G; es decir, con qué finalidad se emprende la talla de un bloque de materia prima. En principio, existen dos objetivos que se denominan:

- Configuración: persigue la obtención de una forma determinada de la BN1G, mediante la extracción de lascas (BN1G c).

- Producción: persigue la obtención de soportes o bases positivas - las BN1G explotadas mediante este sistema son los denominados núcleos- (BN1G p).

Esta diferenciación entre producción y configuración - dèbitage vs façonnage - (Boëda, 1991), no es un criterio directamente observable sino que se deduce a partir de la observación y el análisis de la morfología final de la BN1G.

Los sistemas de configuración (BN1G c) no se analizan directamente en este trabajo. Simplemente se propone un primer nivel de clasificación según el número de superficies de talla que poseen, coincidiendo aproximadamente, con la clasificación más extendida (Bordes, 1979).

A partir de este momento, por tanto, solamente se tratan los sistemas de producción (BN1G p), ya que, debido a sus características, deben ser objeto de un estudio específico.

El primer atributo que se ha utilizado para clasificar los sistemas de explotación es el número de planos en los que se sitúan las extracciones; es decir, el número de superficies de talla. 
De esta manera, aparecen los sistemas de talla unifaciales (UF), cuyas extracciones se sitúan en una sola superficie, que no siempre es plana, sino que puede "envolver» el núcleo. Dentro de este conjunto se encuentran todos los núcleos explotados en una superficie, cuyos máximos exponentes son los núcleos laminares prismáticos.

Lo que, en un segundo nivel, discrimina diferentes tipos de sistemas de talla dentro de los esquemas unifaciales, es la relación geométrica entre la plataforma y la superficie de talla. En los sistemas de explotación unifaciales simples (S), el ángulo entre ambos atributos es menor o próximo a los $45^{\circ}$. Este sería el caso de esquemas de talla sobre lasca tipo Kombewa en su estado de inicio, si solo se ha utilizado una única superficie de talla; es decir, en los casos en que no se haya necesitado un acondicionamiento de la plataforma de talla. También pertenecen a este grupo los núcleos centrípetos unifaciales, denominados discoides en algunas ocasiones (Loch, Swinnen, 1994).
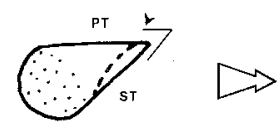

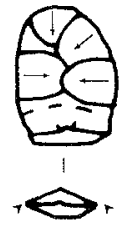

1

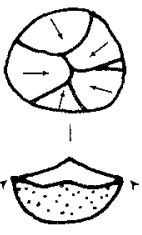

2

Fig. 1. Sistemas unifaciales simples (BN1G p UF A). La relación entre plataforma de talla y superficie de talla es menor o próxima a los $45^{\circ}$ 1: esquema Kombewa sin preparación de la plataforma; 2: discoide unifacial.

Por el contrario, en el caso en que la relación plataforma-superficie se produzca en un ángulo mayor de $45^{\circ}$ y próximo a los $90^{\circ}$ - por encima de lo cual ya no sería posible la talla--, los sistemas de explotación pasarían a denominarse unifaciales abruptos (A). En este grupo se incluyen todas las BN1G de morfología prismática o pseudo-prismática, desde los más simples como los que se han documentado en la Cova de l'Estret de Tragó (Castañeda, Mora, 1999) (fig. 2), con extracciones poco estandarizadas, hasta los más complejos sistemas de explotación laminar. Estos esquemas se caracterizan analíticamente de forma idéntica ya que, en el ámbito conceptual, responden geométricamente al mismo conjunto de atributos y a los mismos principios de talla. Se puede proponer la hipótesis de que una estandarización progresiva del primer sistema de explotación daría lugar al segundo, incluso dentro del mismo contexto del Paleolítico Medio (Révillon, Cliquet, 1994, Kuhn, 1995). 

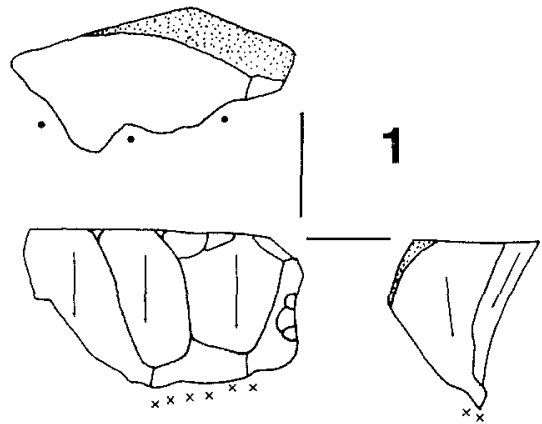

3
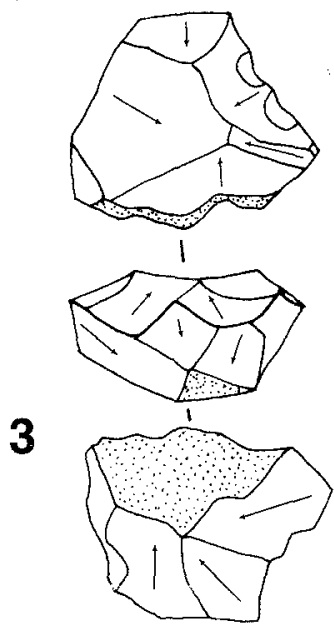

2
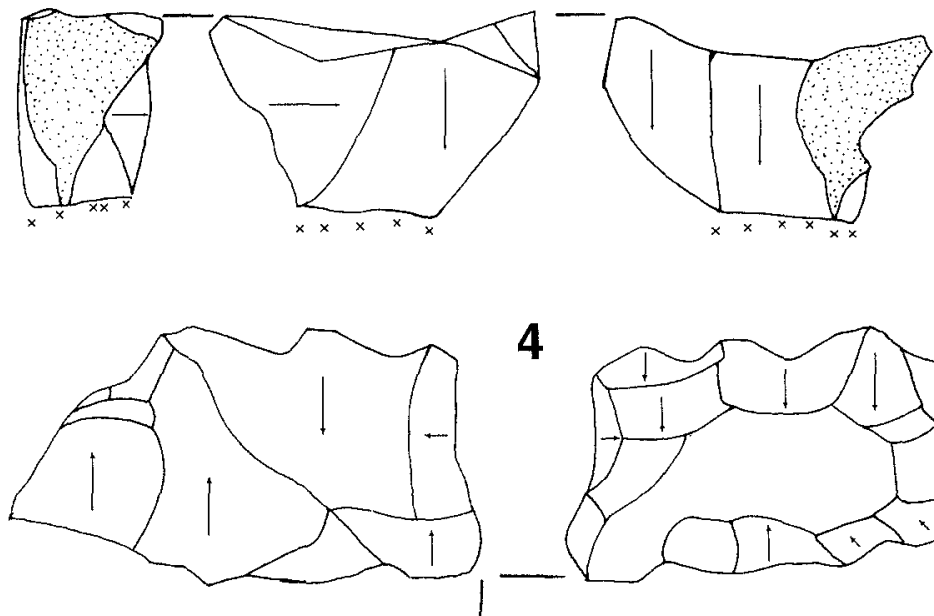

4
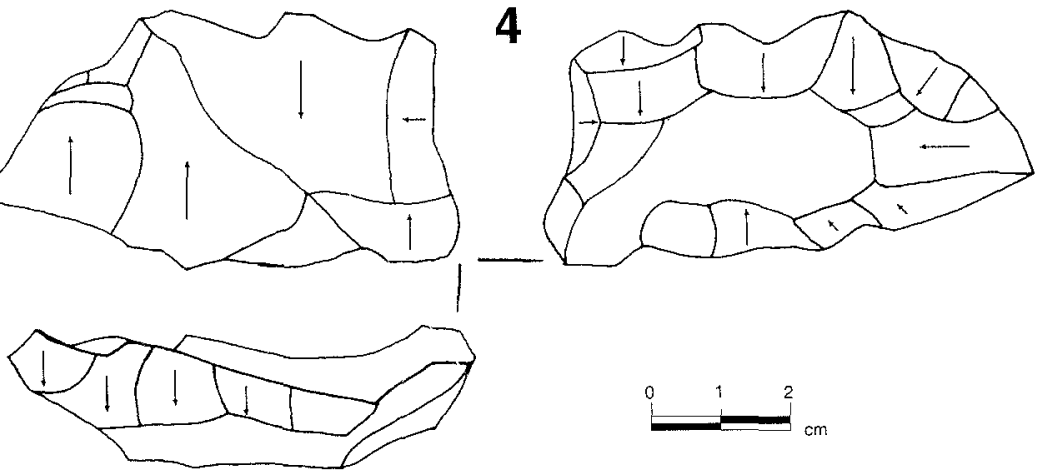

Fig. 2. Algunos ejemplos de BN1G de producción documentados en la Cova de l'Estret de

Tragó (Castañeda, Mora, 1999). 1: Unifacial Abrupto unipolar; 2: Unifacial Abrupto perpendicular; 3: Bifacial Secante No Jerárquico Discoide; 4: Bifacial Secante Jerárquico. 


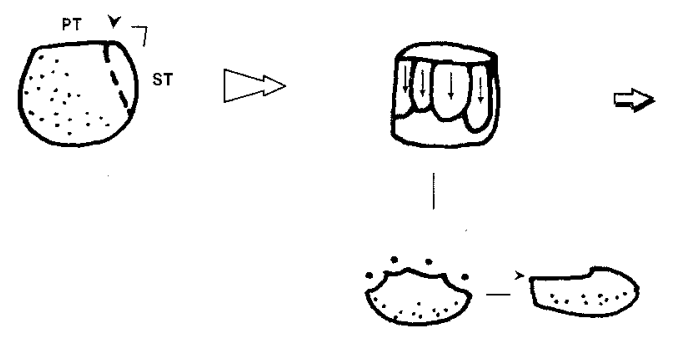

1

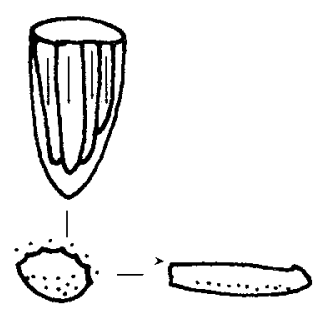

2

Fig. 3. Sistemas unifaciales abruptos (BN1G p UF A). La relación entre plataforma de talla y superficie de talla es mayor de $45^{\circ}$ y próxima a los $90^{\circ}$. 1: esquema prismático o pseudoprismático de lascas; 2: esquema laminar.

El principio básico por el que se rige el sistema unifacial abrupto es la recurrencia en la extracción de productos paralelos. Esto permite mantener la superficie de talla en condiciones aptas para continuar la explotación, sin necesidad de la realización de reacondicionamientos específicos. La plataforma de talla, por otra parte, requiere una preparación muy simple o ausente. La extracción recurrente de productos paralelos secantes, consigue que las aristas se dispongan en la misma dirección en la superficie, permitiendo el mantenimiento de la convexidad necesaria para la talla.

El sistema de explotación unifacial abrupto puede dar lugar a distintos esquemas que siguen el mismo principio. Los caracteres diferenciadores no son de tanta importancia como los que se han utilizado hasta ahora para jerarquizar esta clasificación, ya que estos modelos de explotación distintos son perfectamente compatibles entre sí como para haberse podido emplear durante el proceso de explotación de un mismo núcleo.

De este modo, los sistemas unifaciales abruptos pueden conseguirse a partir de una o dos plataformas de talla. Si la extracción se ha producido a partir de una sola plataforma, el esquema es unipolar (U), y los negativos pueden disponerse rodeando toda la plataforma de talla, solamente en una parte, o en grupos separados en distintas zonas de la plataforma.

En ocasiones se hace necesaria la talla a partir de dos plataformas (B). El grupo de esquemas de talla que se genera de esta forma se caracteriza por la relación existente entre ambas plataformas. En el caso en que éstas sean paralelas entre sí, el esquema se denomina bipolar (bp), si, por el contrario, se disponen en ángulo de $90^{\circ}$ se trata de un esquema perpendicular $(p p)$. 
En el primer caso, bipolar, las aristas paralelas de las extracciones realizadas a partir de la plataforma 1, son aprovechadas para realizar las extracciones desde la plataforma 2. De esta manera, si la talla es alternante el acondicionamiento de la superficie de talla es constante.

En el segundo caso, perpendicular, el agotamiento de la plataforma o de la superficie de talla provoca la búsqueda de nuevas convexidades. Mediante el giro del núcleo en $90^{\circ}$ se encuentra una superficie óptima para iniciar una nueva serie de extracciones paralelas en la misma superficie de talla. Esta convexidad idónea es la arista de interacción de la plataforma de talla 1; es decir, la cornisa que acaba de ser abandonada.

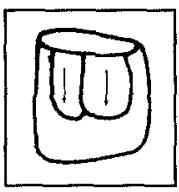

1

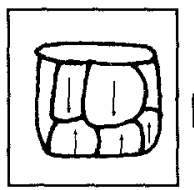

2

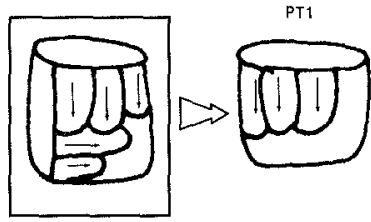

3

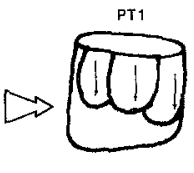

$\Rightarrow$
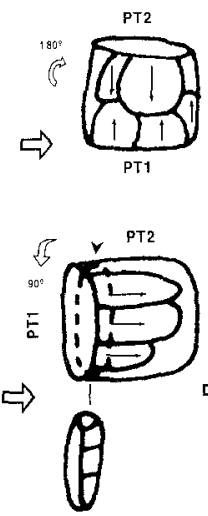

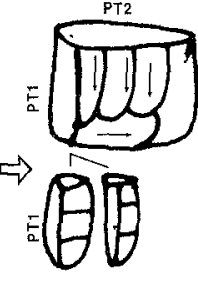

Fig. 4. Modelos de explotación unifacial abrupta (BN1G p UF A). 1: unipolar; 2: bipolar; 3: perpendicular; PT1: plataforma de talla 1; PT2: plataforma de talla 2.

Este sencillo reacondicionamiento de la superficie de talla da lugar a una primera serie de extracciones muy características: primero, la lasca que contiene en su anverso la antigua arista de interacción (fig. 4.3), y posteriormente, productos de caras dorsales con extracciones transversales.

Los sistemas de explotación bifaciales son aquellos en los que las extracciones se distribuyen en dos superficies de talla. En este grupo se puede hacer una distinción importante según la relación que posean ambas superficies de talla:

- Ortogonales $(O)$, en el caso de que ambas superficies formen un diedro próximo a los $90^{\circ}$. En este caso, la explotación de una de las superficies no condiciona la explotación de la otra, es decir, ambas superficies 
son independientes y la arista que tienen en común ambas plataformas no puede considerarse arista de interacción.

- Secantes (S), si los límites de una y otra superficie coinciden en una misma arista. Estos sistemas se basan en el principio de interacción entre ambas superficies de talla. La extracción sobre una de ellas tiene consecuencias importantes para la extracción de productos sobre la otra.

Los sistemas ortogonales se rigen por los mismos criterios que los sistemas unifaciales abruptos, ya que su explotación consiste en la extracción de series paralelas de productos. La recurrencia de este modelo puede dar lugar a la transformación a un sistema unifacial abrupto, si se continúa la talla en una de las superficies hasta eliminar la arista que la separa de la otra. Un ejemplo de este sistema se puede observar en el yacimiento de Beauvais (Oise, Francia) (Loch, Swinnen, 1994).

Los sistemas bifaciales secantes son los más habituales de los sistemas bifaciales y han sido objeto de mayor número de estudios (Boëda, 1993, 1994). En estos modelos, es en la dialéctica que tiene lugar entre ambas superficies de talla, donde se aprecian las diferencias entre estos esquemas de talla: si existe o no una jerarquización en la explotación de ambas superficies.

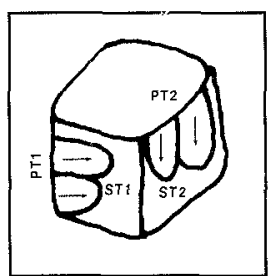

1

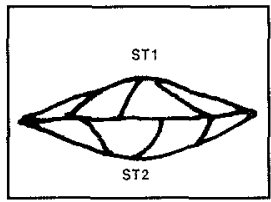

2
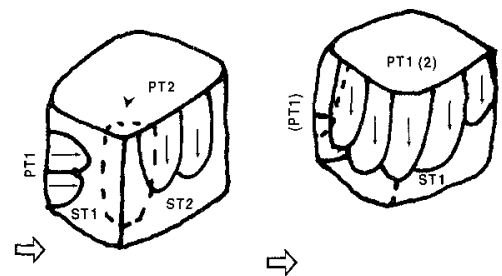

$\Rightarrow$

$\Rightarrow$

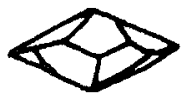

a

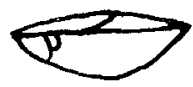

b

Fig. 5. Sistemas de explotación bifacial. 1: sistemas ortogonales, en el transcurso de la explotación pueden transformarse en sistemas unifaciales abruptos; 2; sistemas secantes; a: no jerárquicos; $b$ : jerárquicos. 
Los sistemas jerárquicos poseen una diferenciación entre las funciones de las dos superficies de talla: una primera se utiliza para configurar la plataforma de percusión, y una segunda, preferencial, sobre la que se extraen productos predeterminados.

A través del estudio de remontajes completos se han podido describir los distintos esquemas de preparación de estos núcleos, destinados a producir soportes predeterminados y regularizados. Dichos métodos tienen como resultado productos y núcleos de características técnicas similares. Tanto es así, que existe un consenso ante la imposibilidad de distinguir un esquema de otro a través de las BP o BN1G aisladas. Es necesario para su identificación y análisis, disponer de remontajes en los que se observe el proceso de configuración y explotación prácticamente completo (Van Peer, 1995).

El interés despertado por el método Levallois ha dado lugar a numerosos estudios cada vez más profundos, a través de los cuales se puede caracterizar este modelo de explotación de BN1G bifacial jerárquica. A partir de la definición establecida por E. Boëda (1994), se considera Levallois a la configuración de una superficie de talla preferencial a partir de productos predeterminantes destinados a conseguir dos convexidades laterales y una distal, que proporcionen la morfología idónea para un producto predeterminado. Esta configuración puede mantenerse mediante recurrencia (Levallois recurrente), o mediante sucesivos reacondicionamientos específicos (Levallois de lasca preferencial). De cualquier modo, la preparación y explotación de los núcleos mediante el método Levallois puede realizarse mediante la extracción de lascas en sentido centrípeto, paralelo o bipolar.

Entre los métodos bifaciales jerárquicos no Levallois se encuentran el esquema Victoria West (Merino, 1994), los esquemas Nubio I y Nubio II (Van Peer, 1995), el esquema de los núcleos «libra de mantequilla» (Brézillon, 1983) o la talla Kombewa con preparación de plataforma (Merino, 1994).

Los esquemas bifaciales no jerárquicos no diferencian las superficies de talla, sino que ambas se utilizan para la misma función. En este grupo, se encuentran principalmente los núcleos discoides.

El sistema de explotación no jerárquico discoide (D), consiste en la extracción de productos de manera centrípeta, alternando ambas superficies de talla. Este intercambio recurrente consigue el acondicionamiento constante de las superficies de talla, que mantiene una serie de aristas convergentes y una arista de interacción zigzagueante llena de convexidades alternas sobre las que poder extraer lascas (Boëda, 1993). El resultado de 


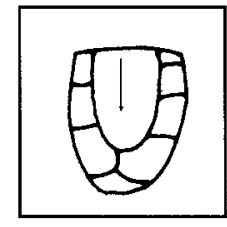

1
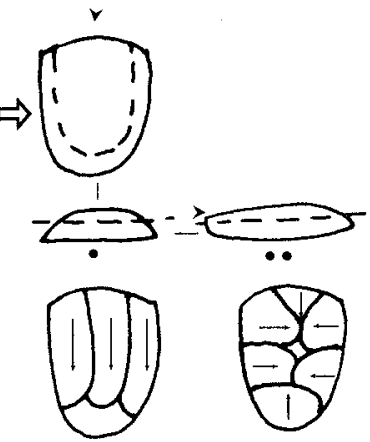

a

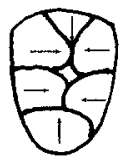

b

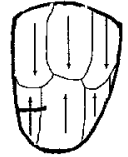

c

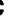

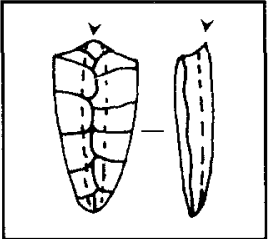

2

Fig. 6. Algunos sistemas de explotación bifacial jerárquica. 1: Levallois, en línea discontinua se señala la extracción predeterminada; $\cdot$ convexidades laterales;

-.: convexidad distal. Dirección de las extracciones predeterminadas: a: paralelo;

b: centrípeto; c: bipolar. 2: núcleo "libra de mantequilla", extracción de láminas mediante la filosofía Levallois.

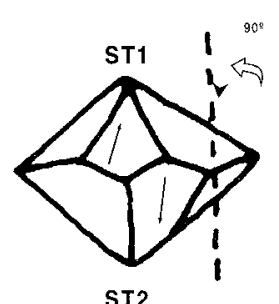

a
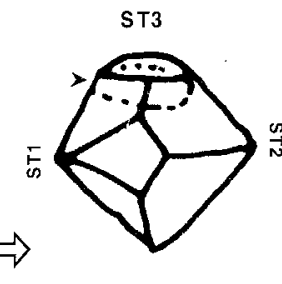

$\Rightarrow$

b

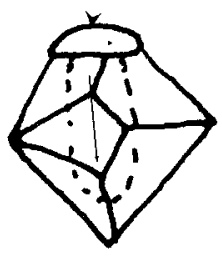

C

Fig. 7. Transformación de un núcleo discoide (a) en un núcleo de explotación trifacial (b) y, posteriormente a uno bifacial ortogonal (c). 
este proceso es un conjunto de productos con talón bifacetado, con tendencia a aumentar en la dimensión anchura frente a la longitud y con un esquema complejo de distribución de negativos en la cara dorsal.

Por último, existen sistemas de explotación para los que son necesarias más de dos superficies de talla: se trata de los sistemas multifaciales. En este grupo se encuentran los núcleos trifaciales y los núcleos irregulares. El primer caso parte de la existencia de tres aristas de interacción y otras tantas superficies de talla. Se han documentado casos de este tipo de explotación a partir de un reavivado de núcleos discoides (Loch, Swinnen, 1994). Una recurrencia en este esquema puede dar lugar a un sistema bifacial ortogonal.

Por último, el sistema irregular, consiste en la explotación de un volumen a partir de diversas plataformas de talla. Mediante este esquema es posible obtener lascas o productos laminares (Brézillon, 1983).

De esta manera, se obtiene un esquema de clasificación de los sistemas de explotación de las BN1G de producción, estructurado en tres niveles principales de discriminación: según el número de superficies de talla, según las interacciones entre atributos, y, por último, según la dirección de la explotación.

Por otra parte, ya se ha comentado que un proceso de explotación de un núcleo es algo muy dinámico que puede dar lugar a cambios en el sistema de explotación empleado, ya que una extracción implica una transformación en las superficies y plataformas de talla. En general, la recurrencia de series paralelas puede invadir y eliminar superficies de talla, y la recurrencia de series centrípetas eliminar elementos de predeterminación, como las convexidades Levallois y puede conseguir la desaparición de la jerarquización de una superficie sobre otra en sistemas bifaciales.

\section{BILIOGRAFÍA}

BAMFORTH, D. (1986): «Technological Efficience and Tool Curation», American Antiquity, 51 (1), págs. $38-50$.

BerTOUILLE, H., (1989): Teories physiques et mathematiques de la taille des outils prehistoriques, Cahiers du Quaternaire, 15, París.

BINFORD, L., (1979): "Organization and iormation processes: looking at curated technologies», Journal of Anthropological Research, 35 (3), págs. 225-273.

BleEd, P., (1986): "The Optimal Design of Hunting Weapons: Maintainability or Reliability", American Antiquity, 50(4), págs. 737-47.

BOËDA, E., (1988): "Le concept laminaire: rupture et filiation avec le concept Levallois», en OTTE, M. (ed.), l'Homme de Neandertal, vol. 5, La Mutation, Liège.

- (1991), «Aproche de la variabilité des systèmes de production lithique des industries du Paléolithique Inférieur et Moyen: chronique d'une variabilité attendue", Techniques et culture, 17-18, págs. 37-78. 


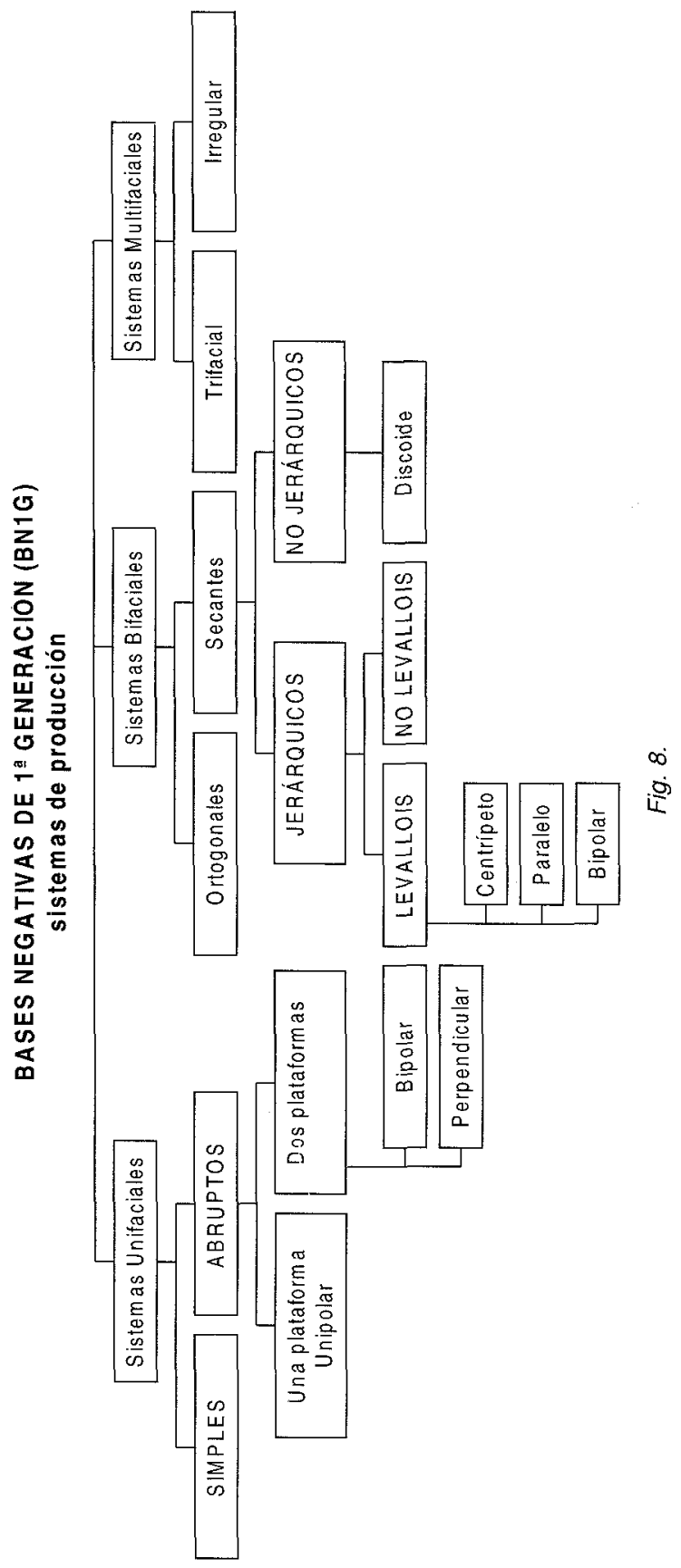


- (1993): «Le débitage discoïde et le débitage Levallois récurrent centripète», Bulletin de la Socièté Préhistorique Française, vol. 90, 6, págs. 393-404.

- (1994): Le concept Levallois: variabilité des méthodes, París, CNRS.

Bordes, F., (1979): Typologie du Paléolithique ancien et Moyen, Cahiers du Quaternaire, 1, CNRS, París.

BRÉZILLON, N., (1983): La dénomination des objects de pierre taillée, CNRS.

CAstAÑEDA, Nuria (1999): La Cova de l'Estret de Tragó. Estrategias de explotación de los recursos minerales en el Paleolítico Medio del NE Peninsular, Trabajo de investigación de inédito presentado en la Universitat Autònoma de Barcelona.

CASTAÑEDA, Nuria; Mora, Rafael (1999): "Un modelo de explotación de los recursos minerales en el Paleolítico Medio: la Cova de l'Estret de Tragó (Lleida)", en PaLlí, L., Rooué, C., (eds.), Avances en el estudio del Cuaternario español. Actas de la X Reunión Nacional de Cuaternario, Girona, págs. 265-270.

CARBONELL, E., GUilbaUd, M., MORA, R., (1983): «Utilización de la lógica analítica para el estudio de tecno-complejos de cantos tallados", Cahier noir, 1, págs. 1-64.

DELAGNES (1995): "Faible élaboration technique et complexité conceptuelle: deux notions complémentaires», Cahier Noir, 7, págs. 101-110.

GalLET, M. (1998): Pour une technologie des débitages laminaires préhistoriques, CNRS, París.

KUHN, S.L. (1995): Mousterian lithic technology. An ecological perspective,Princeton.

LOCH, J.L.; SWINNEN, C. (1994): "Le débitage discoïde du gisement de Beauvais (Oise): aspects de la chaîne opératoire au travers de quelques remontages», Paléo, 6, CNRS, págs. 89-105.

Merino, J.M. (1994): Tipologia lítica, Munibe, Suplemento, 9, San Sebastián.

MorA, R. (1994): «El sistema lógico-analítico» en Merino, J.M., Tipologia lítica, Munibe, Suplemento, 9, San Sebastián, cap. III.

Mora, R.; Martínez, J. Terradas, X. (1992): «Un proyecto de análisis: el Sistema Lógico Analítico (SLA)", en Mora, R., Terradas, X., Parpal, A., Plana, C., (eds.), Tecnología y cadenas operativas liticas, Treballs d'Arqueologia, I, Bellaterra, págs. 173-200.

Nelson, M.C., (1991): "The Study of Technological Organization», en SCHIFfER, M., (ed.), Archaeological Method and Theory, vol. 3, Tucson, págs. 57-100.

PerPéRE, M., (1989): "Les frontières du débitage Levallois: typométrie des éclats", L'Anthropologie, 95 (4), págs. 837-850, París.

RÉVILLion, S., Cliquet, D., (1994): «Technologie du débitage laminaire du gisement paléolithique moyen de Saint Germain-des-Vaux/Port Racine (secteur I) dans le contexte des industries du Paléolithique moyen du Massif Armoricain», en Revillión, S., Tuffreau, A., (eds.), Les industries laminaires au Paléolithique moyen, París, págs. 45-62.

THor, N., (1987): «Behavioral inferences from Early stone artifact assembleges: an experimental model», Journal of Human Evolution, 16, págs. 763-787.

Van Peer, P., (1995): "Current Issues in the Levallois Problem», Dibble, H.L., Bar-Yosef, O., (eds.), The definition and Interpretation of Levallois Technology, Monographs in World Archaeology, 23, Madison, págs. 1-9. 